\title{
Méthodes immunochimiques de dosage de la glycinine et de la $\beta$-conglycinine dans les dérivés du soja. Prédiction de la digestibilité de l'azote et de l'immunogénicité du soja chez le veau
}

\author{
JP Lallès *, HM Tukur**, R Toullec \\ Laboratoire du jeune ruminant, Inra-Ensa, 65, rue de Saint-Brieuc, 35042 Rennes cedex, France
}

(Reçu le 29 août 1996 ; accepté le 5 novembre 1996)

\begin{abstract}
Summary - Immunochemical tests for measuring glycinin and $\beta$-conglycinin concentrations in soyabean products. Predictive value for nitrogen digestibility and soyabean immunogenicity in the calf. Three types of immunochemical tests were compared for assaying the storage globulins, glycinin and $\beta$-conglycinin, in 15 differently processed soyabean products. They included immunoenzymatic tests carried out either on microtitre plates (Elisa) or on nitrocellulose membranes (dot blot), and the passive haemagglutination inhibition test (PHI). These assays also differed in their detection systems: enzymo-colorimetric activity measured spectrophotometrically at $405 \mathrm{~nm}$ (Elisa) or detected visually on membranes (dot blot), and red blood cell agglutination determined spectrophotometrically at $540 \mathrm{~nm}(\mathrm{PHI})$. The hyperimmune sera used in these assays were produced in rabbits against native or SDS-denatured glycinin and native $\beta$-conglycinin. The concentrations of antigens were determined in borate buffer extracts of the soyabean products, and were expressed as $\mathrm{mg} / \mathrm{g}$ of crude protein. The treatments applied to the soyabean products included defatting, steam-heating with or without previous water extraction of oligosaccharides, hot aqueous ethanol denaturation, and proteolysis. Nine of them were introduced into milk replacers which were given to preruminant calves at a level of intake of 58 to $60 \mathrm{~g}$ dry matter per $\mathrm{kg}$ of metabolic weight per day, for approximately 100 days. The soyabean products provided 59 to $72 \%$ of the dietary nitrogen, the remainder coming from skim milk powder or sweet whey supplemented with synthetic amino acids. The apparent digestibility of nitrogen, the live weight gain over the entire experimental periods and the plasma anti-soya antibody titres were measured. The data obtained by the different tests for each globulin were highly and positively correlated to one another, even though the dot blot tests for the native globulins provided significantly higher results $(P<0.05)$. The apparent digestibility of nitrogen, measured for the diet or calculated for the soya products, was highly and positively correlated with the concentrations of immunoreactive globulins, for the vast majority of the compared immunochemical assays. Conversely, the plasma anti-soya antibody titres and the levels of immunoreactive globulins varied in the
\end{abstract}

\footnotetext{
* Correspondance et tirés à part.

** Nouvelle adresse : Department of Animal Sciences, Faculty of Agriculture, University of Sokoto, PMB 2346, Sokoto, Nigeria.
} 
same way, irrespective of the assay format. In contrast, the relationships between live weight gain and the levels of immunoreactive globulins in the products were rarely significant. Finally, the relationships between in vivo and in vitro parameters, when significant, were the strongest with $\beta$-conglycinin. It is concluded from these results that all these assays employing hyperimmune sera raised against the native soyabean globulins for determining the levels of antigenic proteins in soyabean products intended to be incorporated into milk replacers are useful because they all could predict the apparent digestibility of dietary or soyabean nitrogen, and the immunogenicity of soyabean in the preruminant calf. Elisa remains the method of choice in the laboratory conditions, while the PHI and the dot blot assays could be used when the technical environment is not appropriate for Elisa.

\section{calf / digestion / immunochemistry / protein / soyabean}

Résumé - Trois types de techniques immunochimiques ont été comparées pour doser la glycinine et la $\beta$-conglycinine dans 15 dérivés du soja. Il s'agissait soit de tests immunoenzymatiques sur plaques (Elisa) ou sur membrane (immunotache), soit d'inhibition de l'hémagglutination passive de globules rouges (IHP). La quantification était basée soit sur une réaction enzymocolorométrique mesurée à $405 \mathrm{~nm}$ (Elisa) ou appréciée visuellement (immunotache), soit sur une agglutination détectée à $540 \mathrm{~nm}$ (IHP). Les sérums hyperimmuns utilisés étaient dirigés contre la glycinine native ou dénaturée et contre la $\beta$-conglycinine native. Les résultats obtenus par les trois types de techniques ont été très corrélés positivement entre eux. Toutefois, l'immunotache a fourni des valeurs significativement plus élevées que l'Elisa et l'IHP. Neuf des 15 dérivés du soja ont été incorporés dans des aliments d'allaitement distribués à des veaux préruminants. La digestibilité apparente de l'azote et le titre d'anticorps plasmatiques anti-soja ont été fortement corrélés, respectivement négativement et positivement, aux teneurs en glycinine et $\beta$-conglycinine immunoréactives obtenues par la majorité des tests in vitro. Les relations ont été les plus fortes avec la $\beta$-conglycinine. En revanche, les relations ont rarement été significatives pour le gain de poids vif.

\section{digestion / immunochimie / protéines / soja / veau}

\section{INTRODUCTION}

Le soja est une source importante de protéines en alimentation animale. Il contient cependant des facteurs antinutritionnels dont les activités des plus connus, les inhibiteurs trypsiques et les lectines, sont largement réduites par chauffage (revue de Huisman, 1989). De plus, les globulines de réserve, la glycinine et la $\beta$-conglycinine, qui représentent les deux tiers des protéines de la graine (Murphy et Resurreccion, 1984), sont partiellement résistantes aux traitements thermiques et à la digestion par les protéases de mammifères (Nielsen et al, 1988 ; Sissons et Thurston, 1984). Ces protéines sont immunogènes chez le veau préruminant, c'est-à-dire qu'elles induisent la formation d'anticorps spécifiques, lorsqu'elles sont incorporées dans les aliments d'allaitement sous forme insuffisamment dénaturée ; elles sont alors suspectées de perturber la physiologie digestive par des mécanismes immunitaires de nature allergique (revues de Sissons, 1982 ; Lallès et Toullec, 1996).

Les concentrations de protéines antigéniques dans les produits du soja ont été estimées semi-quantitativement par inhibition de l'hémagglutination passive (IHP) puis par dosage immunoenzymatique (Elisa), à l'aide de sérums hyperimmuns produits chez le lapin contre les antigènes peu ou pas dénaturés (Kilshaw et Sissons, 1979 ; Heppell et al, 1987). Les résultats étaient exprimés sous forme de titre, c'est-à-dire par un nombre de dilutions au demi d'un extrait de soja donné, au-delà duquel l'hémagglutination de globules rouges de mouton apparaissait dans le cas de l'IHP, ou au-delà ou en deçà duquel la densité optique (DO) de l'échantillon était deux fois supérieure à la 
DO correspondant au bruit de fond, selon le type de test Elisa pratiqué. Dans ces conditions, il était très difficile de comparer les résultats entre laboratoires. Ces méthodes ont néanmoins permis, d'une part, d'optimiser les conditions de réduction de l'immunoréactivité du soja par dénaturation en milieu hydro-éthanolique à chaud (Sissons et al, 1982), et, d'autre part, de démontrer que de la glycinine et de la $\beta$-conglycinine immunoréactives échappaient à la digestion chez le veau (Sissons et Thurston, 1984).

Nous avons récemment montré que, lorsque les activités antigéniques sont mesurées quantitativement ( $\mathrm{en} \mathrm{mg} / \mathrm{g}$ de matières azotées) par Elisa, elles peuvent servir à établir des équations de prévision de la digestibilité apparente fécale de l'azote du soja (Toullec et al, 1994b ; Lallès et al, 1996b). Il est toutefois déterminant de réaliser les dosages immunochimiques en employant des sérums hyperimmuns spécifiques dirigés contre les globulines natives. En effet, l'utilisation de certains anticorps monoclonaux actuellement disponibles (Carter et al, 1992 ; Plumb et al, 1994, 1995) ne permet pas de prévoir la digestibilité de l'azote du soja ou son immunogénicité (Tukur et al, 1996).

L'objet du présent travail est de vérifier que ces sérums hyperimmuns spécifiques peuvent être utilisés dans des techniques immunochimiques quantitatives, différentes de l'Elisa, à savoir l'IHP et l'immunotache, tout en conservant une qualité de prédiction de la digestibilité de l'azote du soja. Une partie de ces travaux a été publiée sous forme de communications (Tukur et al, 1994 ; Lallès et al, 1995a).

\section{MATÉRIEL ET MÉTHODES}

\section{Produits issus du soja}

Les tests immunochimiques ont porté sur 15 produits issus du soja : trois farines crues délipidées (FD), quatre farines délipidées et chauffées (FC), trois concentrats extraits à l'eau puis chauffés
(CEC), deux concentrats extraits en milieu hydroéthanolique à chaud (CEAC) et trois concentrats extraits à l'eau et partiellement protéolysés (CEPP) (tableau I). Les produits FC4, CEC3 et CEPP2 ont subi des traitements supplémentaires non dévoilés par les fabricants. Tous ces produits ont été préparés industriellement (Toullec et al, 1994a ; Lallès et al, 1995b, c ; Tukur et al, 1995), excepté deux farines (FD1 et FD2) qui ont été délipidées au laboratoire à l'aide d'éther de pétrole, à température ambiante. La composition détaillée a été rapportée antérieurement (Toullec et al, 1994a ; Lallès et al, 1995b, c ; Tukur et al, 1995 ; Lallès et al, 1996b). À l'exception des farines crues, tous les produits ont été incorporés dans des aliments d'allaitement pour veaux. Cependant, trois séries de résultats in vivo n'ont pas été prises en compte ici car deux produits (FC3 et CEAC2) ont servi dans une étude de digestibilité iléale et non fécale, et le troisième (CEPP3) s'est révélé être un mélange de protéines de soja hydrolysées et de lactosérum (proportions de 75 et $25 \%$, sur la base des matières azotées [MA : $\mathrm{N} \times 6,25]$ et d'acides aminés de synthèse (Lallès et al, 1995c).

\section{Purification des globulines du soja et préparation des sérums hyperimmuns}

Les techniques de purification des globulines du soja et de préparation des sérums hyperimmuns ont été décrites par Tukur et al (1993). La glycinine dénaturée a été obtenue par chauffage $\left(100{ }^{\circ} \mathrm{C}\right.$ pendant 3 minutes) en présence de dodécyl sulfate de sodium (SDS) I \% dans du tam-

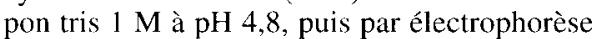
sur gel de polyacrylamide en conditions réductrices, afin de séparer les polypeptides acides et les polypeptides basiques d'éventuels contaminants mineurs (Tukur et al, 1993). Les portions de gel contenant chacun des polypeptides ont été découpées puis mélangées ensemble et à de l'adjuvant, pour être finalement injectées à des lapins selon le protocole décrit par Boulard et Lecroisey (1982).

\section{Dosages immunochimiques}

Les divers types de dosages immunochimiques étudiés sont présentés succinctement dans le tableau II. Ils ont été réalisés sur des extraits des produits du soja en tampon borate $0,1 \mathrm{M}$ à pH 8,0 (Tukur et al, 1993). 
Tableau I. Dérivés du soja étudiés et concentrations individuelles et médianes ( $\mathrm{g} / 100 \mathrm{~g} \mathrm{MA})$ de glycinine immunoréactive.

$\begin{array}{cc}\text { Type de dosage } & \\ \text { Elisa } & \text { Inhibition de } \\ \text { l'hémagglutination } & \text { passive }\end{array}$ Immunotache

\section{Sérum hyperimmun}

contre l'antigène

Natif

Natif

Dénaturé

Natif

Dénaturé

Farines délipidées

$\begin{array}{llllrl}\text { FD1 } & 12,4 & 21 & 21 & 21 & 21 \\ \text { FD2 } & 26,9 & 29,3 & 29,3 & 147 & 58,7 \\ \text { FD3 } & 28,7 & 24,8 & 24,8 & 124 & 24,8\end{array}$

Farines chauffées

$\begin{array}{llllll}\mathrm{FC}^{1}{ }^{1} & 3,9 & 3 & 3 & 30 & 3 \\ \mathrm{FC}^{1}{ }^{1} & 2,7 & 5,1 & 5,1 & 12,7 & 2,5 \\ \text { FC }^{1} & 1,7 & 4,1 & 4,2 & 10,4 & 2,1 \\ \text { FC4 }^{1} & 0,1 & 0,1 & 0,05 & 3,9 & 0,4\end{array}$

Concentrats extraits à l'eau et chauffés

$\begin{array}{llllrl}\text { CEC }^{1} & & & & \\ \text { CEC2 }^{1} & 2,0 & 2,3 & 1,1 & 2,8 & 2,3 \\ \text { CEC }^{1} & 3,3 & 2 & 1 & 10,2 & 4,1 \\ & 0,003 & 0,03 & 0,004 & 0,15 & 0,23\end{array}$

Concentrats extraits dans un mélange d'eau et d'alcool chaud

$\begin{array}{llllll}\text { CEAC }^{1} & 0 & 0,04 & 0,003 & 0,18 & 0,14 \\ \text { CEAC2 } & 0 & 0,03 & 0,0002 & 0,12 & 0,1\end{array}$

Concentrats extraits à l'eau et partiellement protéolysés

\begin{tabular}{llllll} 
CEPP $1^{1}$ & 0 & 0 & 0 & 0 & 0 \\
CEPP2 ${ }^{1}$ & 1,1 & 0,49 & 0,06 & 4,9 & 2 \\
CEPP3 & 0 & 0 & 0,002 & 1,5 & 1,2 \\
Médiane $(n=15)$ & $1,7^{\mathrm{a}}$ & $2^{\mathrm{a}}$ & $1^{\mathrm{a}}$ & $4,9^{\mathrm{b}}$ & $2,1^{\mathrm{ab}}$ \\
Médiane $(n=9)^{\mathrm{l}}$ & $1,1^{\mathrm{a}}$ & $0,49^{\mathrm{ab}}$ & $0,06^{\mathrm{ab}}$ & $3,9^{\mathrm{b}}$ & $2^{\mathrm{ab}}$ \\
\hline
\end{tabular}

1 Produits utilisés dans les études in vivo. ${ }^{2}$ Pour plus de détails, consulter le tableau II. ${ }^{\mathrm{a}, \mathrm{b}}$ Les valeurs médianes d'une même ligne accompagnées de lettres différentes sont significativement différentes $(p<0,05)$.

Les tests Elisa destinés à doser la glycinine et la $\beta$-conglycinine natives sont ceux décrits par Tukur et al (1993). Brièvement, des plaques de dosage de 96 puits à fond plat ont été recouvertes de l'une ou l'autre des globulines purifiées. Pendant ce temps, diverses dilutions au demi des globulines purifiées (gammes) ou des extraits de produits du soja (échantillons) ont été mélangées à une quantité fixe de l'un des sérums hyperimmuns spécifiques dilués. Le lendemain, les plaques de dosage ont été lavées puis saturées, et les mélanges décrits ci-dessus déposés sur ces plaques. Après incubation puis lavage, une solution d'anticorps anti-immunoglobulines de lapin 
Tableau II. Dosages immunochimiques développés pour la glycinine et la $\beta$-conglycinine natives.

\begin{tabular}{lcc}
\hline Antigène & Type de dosage & Spécificité du sérum hyperimmun \\
\hline Glycinine & Elisa $^{2}$ & Antigène natif \\
& $\mathrm{IHP}^{3}$ & Antigène natif \\
& $\mathrm{IHP}^{3}$ & Antigène dénaturé par chauffage en présence de SDS \\
& Immunotache $^{4}$ & Antigène natif \\
$\beta$-conglycinine & Immunotache & Antigène dénaturé par chauffage en présence de SDS \\
& Elisa $^{2}$ & Antigène natif \\
& IHP $^{3}$ & Antigène natif \\
& Immunotache & Antigène natif
\end{tabular}

${ }^{1}$ L'antigène déposé sur les plaques Elisa, sur les globules rouges de mouton traités (IHP) ou sur les membranes de nitrate de cellulose a été, dans tous les cas, sous forme native. ${ }^{2}$ Tukur et al (1993). ${ }^{3}$ Inhibition de l'hémagglutination passive. ${ }^{4}$ SDS : dodécyl-sulfate de sodium $1 \%$, chauffage pendant $3 \mathrm{~min}$.

couplé à la peroxydase a été ajoutée. Après incubation puis lavage, le substrat de l'enzyme (ABTS, en présence d' $\mathrm{H}_{2} \mathrm{O}_{2}$ ) a été ajouté. Les DO des produits solubles générés par la réaction enzymatique ont été mesurées à $405 \mathrm{~nm}$, à l'aide d'un lecteur de plaques (Multiskan, modèle Argus 300, Packard Instruments Co, États-Unis). Les gammes ont servi à déterminer les concentrations de globulines dans les échantillons (Tukur et al, 1993).

Les tests d'IHP ont été effectués à l'aide de globules rouges de mouton, selon la technique décrite par Kilshaw et Sissons (1979). Brièvement, les cellules préalablement traitées à l'acide tannique ont été recouvertes de l'une ou l'autre des globulines purifiées (tableau II), à raison de $25 \mu \mathrm{g}$ de protéines par millilitre de tampon. Diverses dilutions au demi des globulines purifiées ou des extraits des produits du soja à doser ont été mélangées à une quantité fixe de l'un des sérums hyperimmuns spécifiques dilués, sur des plaques de 96 puits à fond en V. Ensuite, un volume fixe de suspension de cellules recouvertes de globulines a été ajouté. Après mélange et incubation nécessaire à l'hémagglutination, la DO a été mesurée à $540 \mathrm{~nm}$, longueur d'onde d'absorption de l'hémoglobine, en trois points équidistants du centre et de la périphérie de chaque puits, à l'aide du lecteur de plaques décrit ci-dessus. L'hémagglutination a été considérée positive dès que la $\mathrm{DO}$ a été supérieure à 0,5 , et le titre déterminé en comptant le nombre de dilutions au-delà duquel l'hémagglutination appa- raissait. Ces conditions de lecture ont été préalablement calées sur des résultats de détermination visuelle des titres d'hémagglutination (JP Lallès et HM Tukur, résultats non publiés). Les concentrations de globulines dans les échantillons ont été calculées à partir des titres d'agglutination des globulines purifiées de concentration connue, des titres d'agglutination propres aux échantillons et des dilutions préalables éventuelles.

Les tests d'immunotache ont été pratiqués à température ambiante selon les procédés généraux habituels (revue de Stott, 1989). Brièvement, des membranes de nitrate de cellulose (Hybon-C super, Amersham, Royaume-Uni) humidifiées ont été placées dans un appareil à immunotaches (Convertible Manifold System, Life Technologies Inc, États-Unis), laissant libres 96 disques accessibles pour déposer, sous vide, les solutions à analyser. Diverses dilutions au demi des globulines purifiées et d'extraits de produits du soja ont été déposées $(5 \mu \mathrm{L})$ sur ces disques. Après lavage puis saturation, les membranes ont été replacées dans l'appareil, et un volume fixe d'un des sérums hyperimmuns dilués a été déposé sur chaque disque. Après lavage, les membranes ont été incubées dans des petits sacs en plastique avec l'anticorps anti-immunoglobulines de lapin couplé à la péroxydase puis, après lavage, avec le substrat de l'enzyme (diamino-benzidine et $\mathrm{H}_{2} \mathrm{O}_{2}$ ). Celui-ci génère un produit insoluble marron qui se dépose sur la membrane, traduisant finalement la présence de la globuline recherchée. Les dilutions au-delà 
desquelles l'immunomarquage disparaissait ont été déterminées visuellement pour les gammes et les échantillons, et les calculs effectués comme dans le cas de l'IHP.

\section{Mesures in vivo}

Les neuf aliments d'allaitement contenant l'un des dérivés du soja retenus pour les mesures in vivo ont été distribués pendant environ $100 \mathrm{j}$ à des veaux préruminants ( $n=5$ à 7 par traitement) âgés initialement de 1 mois (Toullec et al, 1994a ; Lallès et al, 1995b, c ; Tukur et al, 1995). Dans ces aliments, le soja apportait de 58 à $72 \%$ des MA, le reste provenant de poudre de lait écrémé ou de lactosérum et d'acides aminés de synthèse. Les teneurs en MA et en lipides des régimes ont été comprises respectivement entre 20 et $22 \%$, et 18 et $20 \%$ de la matière sèche (MS). Les aliments d'allaitement ont été distribués en deux buvées quotidiennes, à raison de 58 à $60 \mathrm{~g}$ de MS par kg de poids vif ${ }^{0,75}$. La digestibilité apparente fécale de l'azote des régimes (DANR) a été mesurée de façon classique sur une période de $5 \mathrm{j}$; celle du soja (DANS) a été calculée en tenant compte du taux d'incorporation de chaque produit et en supposant que la digestibilité de l'azote de la poudre de lait écrémé et/ou du lactosérum était identique à celle déterminée avec le régime témoin de chaque expérience (Toullec et al, 1994a ; Lallès et al, 1995b, c ; Tukur et al, 1995). Dans chaque étude, le gain de poids vif (GPV) a été calculé durant la période expérimentale complète et rapportée à la valeur observée dans le groupe des veaux témoins qui ne consommaient que des protéines d'origine laitière. Les titres d'anticorps anti-soja (TAAS) ont été déterminés en cinétique dans le plasma, par hémagglutination passive (Kilshaw et Sissons, 1979). Les valeurs retenues dans les présents calculs ont été celles enregistrées à la fin des essais, corrigées des valeurs observées dans les groupes témoins.

En résumé, les valeurs médiane, inférieure et supérieure ont été de $0,88,0,73$ et 0,93 pour la DANR, de $0,76,0,59$ et 0,84 pour la DANS, de 83,73 et $93 \%$ pour le GPV, et enfin de $2,2,-0,1$ et 7,9 pour les TAAS (exprimé en $\log _{2}$ des dilutions des plasmas, à partir de dilutions initiales au 1/20) (Tukur et al, 1996).

\section{Analyses statistiques}

Les concentrations de glycinine et de $\beta$-conglycinine dans les dérivés du soja ont été rapportées aux MA. Les données n'étant pas distribuées normalement, les valeurs médianes ont été indiquées dans les tableaux I et III. Les comparaisons entre méthodes in vitro ont été effectuées

Tableau III. Matrice des coefficients de corrélation des rangs de Spearman entre les concentrations de glycinine immunoréactives déterminées par les divers types de dosages immunochimiques ${ }^{1,2}$.

\begin{tabular}{lcccc}
\hline & $\begin{array}{c}\text { Elisa/SH } \\
\text { anti-GN }\end{array}$ & $\begin{array}{c}\text { IHP/SH } \\
\text { anti-GN }\end{array}$ & $\begin{array}{c}\text { IHP/SH } \\
\text { anti-GD }\end{array}$ & $\begin{array}{c}I T / S H \\
\text { anti-GN }\end{array}$ \\
\hline Elisa/SH anti-GN & - & & & \\
IHP/SH anti-GN & $(-)$ & & & \\
IHP/SH anti-GD & $0,93^{\mathrm{b}}$ & & & \\
IT/SH anti-GN & $\left(0,89^{a}\right)$ & & & \\
IT/SH anti-GD & $0,94^{\mathrm{b}}$ & $0,99^{\mathrm{b}}$ & & \\
& $\left(0,91^{b}\right)$ & $\left(0,98^{b}\right)$ & & \\
& $0,92^{\mathrm{b}}$ & $0,93^{\mathrm{b}}$ & $0,94^{\mathrm{b}}$ & \\
& $\left(0,90^{\mathrm{b}}\right)$ & $\left(0,88^{\mathrm{a}}\right)$ & $\left(0,87^{a}\right)$ & \\
& $0,96^{\mathrm{b}}$ & $0,90^{\mathrm{b}}$ & $0,93^{\mathrm{b}}$ & $0,93^{\mathrm{c}}$ \\
& $\left(0,98^{b}\right)$ & $\left(0,87^{\mathrm{a}}\right)$ & $\left(0,88^{a}\right)$ & $\left(0,88^{b}\right)$ \\
\hline
\end{tabular}

${ }^{1}$ Quinze produits (les valeurs entre parenthèses correspondent aux neuf produits étudiés in vivo). ${ }^{2} \mathrm{SH}$ : sérum hyperimmun, GN : glycinine native, GD : glycinine dénaturée, IHP : inhibition de l'hémagglutinatin passive, IT : immunotache. ${ }^{\mathrm{a}} p<0,01,{ }^{\mathrm{b}} p<0,001$. 
par le test de Friedman, analyse de variance non paramétrique à une voie (Hollander et Wolfe, 1973). Les coefficients de corrélation entre les méthodes de dosage et avec les données in vivo ont été déterminés par le test des rangs de Spearman (Dagnélie, 1970).

\section{RÉSULTATS}

\section{Glycinine immunoréactive}

La concentration de glycinine immunoréactive a été la plus élevée dans les farines crues délipidées (tableau I). Le chauffage, précédé ou non d'une extraction des oligosides à l'eau, a réduit l'immunoréactivité de 80 à $90 \%$. Les traitements plus élaborés, tels que la dénaturation en milieu hydroéthanolique et la protéolyse, ont été plus efficaces et ont le plus souvent rendu les produits très peu antigéniques. En effet, avec ces traitements, et si l'on se réfère à l'Elisa, l'immunoréactivité glycinine résiduelle a été inférieure respectivement à 0,4 et $4 \%$ de celle mesurée dans les produits crus délipidés et dans les produits cuits les plus courants ; l'immunoréactivité $\beta$-conglycinine résiduelle a été inférieure à la limite de détection du dosage.

Parmi les méthodes de dosage étudiées, seule l'immunotache pratiquée avec le sérum hyperimmun anti-glycinine native a donné des valeurs significativement plus élevées $(p<0,05)$ que les autres. Quoi qu'il en soit, toutes ces valeurs ont été très fortement et positivement corrélées entre elles et à celles obtenues par Elisa, généralement considérée comme méthode de référence $(p<0,001 ;$ tableau III).

\section{$\beta$-conglycinine immunoréactive}

Les effets des traitements sur la concentration de $\beta$-conglycinine (tableau IV) ont été voisins de ceux décrits pour la glycinine, exception faite du produit CEPP2. Ici aussi, l'immunotache pratiquée avec le sérum hyperimmun anti- $\beta$-conglycinine native a conduit à des valeurs significativement plus élevées $(p<0,05)$ que celles obtenues par Elisa ou IHP. Toutefois, ces valeurs ont été très fortement et positivement corrélées entre elles $(p<0,001 ;$ tableau V).

\section{Relation entre paramètres in vivo et activités antigéniques in vitro}

Comme nous l'avons déjà mentionné (Tukur et al, 1996), les digestibilités de l'azote des régimes (DANR) et du soja (DANS) ont été négativement corrélées ( $p<0,05$ à $p<0,001$ ) aux concentrations de glycinine et de $\beta$-conglycinine immunoréactives dans les produits, déterminées par Elisa (tableau VI). La même tendance, bien que non significative, a été observée dans le cas du gain de poids vif. En revanche, l'immunogénicité a été, comme on s'y attendait, positivement corrélée à l'antigénicité déterminée par Elisa.

Les même types de relations entre paramètres in vivo et antigénicité in vitro ont été observés dans le cas de l'IHP et de l'immunotache, ce qui est logique compte tenu des corrélations positives élevées entre les méthodes in vitro. Il est toutefois intéressant de remarquer que les corrélations ont été faibles et rarement significatives dans le cas de l'immunotache utilisant le sérum hyperimmun anti-glycinine native. Inversement, ces corrélations ont toujours été significatives ( $p<0,05$ à $p<0,01), y$ compris pour le GPV, dans le cas de la $\beta$ conglycinine déterminée par IHP. Enfin, les coefficients de corrélation les plus élevés ont été observés entre la DANR et la $\beta$ conglycinine détectée par immunotache puis, à égalité, entre la DANR et la glycinine dosée par IHP en présence du sérum hyperimmun anti-glycinine dénaturée, et entre la DANS et la $\beta$-conglycinine dosée par Elisa. 
Tableau IV. Concentrations individuelles et médianes (g/100g MA) de $\beta$-conglycinine immunoréactive dans les dérivés du soja ${ }^{1}$, déterminées à l'aide d'un sérum hyperimmun anti- $\beta$-conglycinine native.

\begin{tabular}{|c|c|c|c|}
\hline & \multicolumn{3}{|c|}{ Type de dosage ${ }^{2}$} \\
\hline & Elisa & $\begin{array}{c}\text { Inhibition de } \\
\text { l'hémagglutination } \\
\text { passive }\end{array}$ & Immunotache \\
\hline \multicolumn{4}{|l|}{ Farines délipidées } \\
\hline FD1 & 9 & 10,5 & 21 \\
\hline FD2 & 15,5 & 14,7 & 58,7 \\
\hline FD3 & 19,4 & 12,4 & 24,8 \\
\hline \multicolumn{4}{|l|}{ Farines chauffées } \\
\hline $\mathrm{FC}^{1}$ & 3,6 & 3 & 6 \\
\hline $\mathrm{FC} 2^{1}$ & 1,3 & 1,3 & 2,5 \\
\hline $\mathrm{FC} 3$ & 2,9 & 2,1 & 4,2 \\
\hline $\mathrm{FC}^{1}{ }^{\mathrm{I}}$ & 0 & 0 & 0,2 \\
\hline \multicolumn{4}{|c|}{ Concentrats extraits à l'eau et chauffés } \\
\hline $\mathrm{CECl}^{1}$ & 2,5 & 1,1 & 4,5 \\
\hline $\mathrm{CEC} 2^{1}$ & 1,5 & 1,0 & 4,1 \\
\hline $\mathrm{CEC} 3^{1}$ & 0 & 0 & 0,23 \\
\hline \multicolumn{4}{|c|}{ Concentrats extraits dans un mélange d'eau et d'alcool chaud } \\
\hline $\mathrm{CEAC}^{1}$ & 0 & 0 & 0,14 \\
\hline CEAC2 & 0 & 0 & 0,1 \\
\hline \multicolumn{4}{|c|}{ Concentrats extraits à l'eau et partiellement protéolysés } \\
\hline CEPP $1^{1}$ & 0 & 0 & 0 \\
\hline CEPP $2^{1}$ & 0 & 0 & 2 \\
\hline CEPP3 & 0 & 0 & 1,2 \\
\hline Médiane $(n=15)$ & $1,3^{\mathrm{ab}}$ & $1^{a}$ & $2,5^{b}$ \\
\hline Médiane $(n=9)^{1}$ & $0^{\mathrm{a}}$ & $0^{a}$ & $2^{a}$ \\
\hline
\end{tabular}

${ }^{1}$ Produits utilisés dans les études in vivo. ${ }^{2}$ Pour plus de détails, consulter le tableau II. ${ }^{\text {a, b}}$ Les valeurs médianes d'une même ligne accompagnées de lettres différentes sont significativement différentes $(p<0,05)$.

\section{DISCUSSION}

Le soja est, après le lactosérum, la première source de protéines de remplacement dans les aliments d'allaitement pour veaux. Il est disponible sous forme de farines élaborées par délipidation à partir de graines décorti- quées, de concentrats obtenus par extraction des oligosides à l'eau ou en milieu hydroéthanolique, et d'isolats préparés par précipitation sélective puis resolubilisation des protéines. Les teneurs respectives en MA de ces trois catégories de produits sont voisines de 50 à $55 \%, 65$ à $85 \%$, et $90 \%$ de 
Tableau V. Matrice des coefficients de corrélation des rangs de Spearman entre les concentrations de $\beta$-conglycinine immunoréactive déterminées par les divers types de dosages immunochimiques, à l'aide d'un sérum hyperimmun anti- $\beta$-conglycinine native' .

\begin{tabular}{lcc} 
& Elisa & IHP \\
\hline Elisa & $(-)$ & \\
& $0,98^{b}$ & \\
Inhibition de l'hémagglutination passive (IHP) & $\left(0,94^{a}\right)$ & $0,93^{b}$ \\
& $0,94^{b}$ & $\left(0,86^{a}\right)$ \\
Immunotache & $\left(0,91^{b}\right)$ & \\
&
\end{tabular}
${ }^{\mathrm{h}} p<0,001$.

Tableau VI. Matrice des coefficients de corrélation de Spearman entre les paramètres in vivo et les concentrations de glycinine ou de $\beta$-conglycinine immunoréactives dans les produits du soja.

\begin{tabular}{|c|c|c|c|c|}
\hline & \multicolumn{4}{|c|}{ Paramètres in vivo' } \\
\hline & $D A N R$ & DANS & $G P V$ & TAAS \\
\hline Elisa/SH ${ }^{2}$ anti-glycinine native & $-0,88^{b}$ & $-0,75^{a}$ & $-0,59$ & $0,82^{b}$ \\
\hline IHP/SH anti-glycinine native & $-0,87^{b}$ & $-0,78^{a}$ & $-0,46$ & $0,77^{a}$ \\
\hline IHP/SH anti-glycinine dénaturée & $-0,90^{\mathrm{c}}$ & $-0,78^{a}$ & $-0,52$ & $0,82^{b}$ \\
\hline Immunotache/SH anti-glycinine native & $-0,73^{a}$ & $-0,61$ & $-0,45$ & 0,62 \\
\hline Immunotache/SH anti-glycinine dénaturée & $-0,83^{\mathrm{b}}$ & $-0,71^{a}$ & $-0,53$ & 0,75 : \\
\hline Elisa/SH anti- $\beta$-conglycinine native & $-0,89^{b}$ & $-0,90^{\mathrm{c}}$ & $-0,66$ & $0,89^{b}$ \\
\hline IHP/SH anti- $\beta$-conglycinine native & $-0,89^{b}$ & $-0,89^{b}$ & $-0,75^{a}$ & $0,89^{b}$ \\
\hline Immunotache/SH anti- $\beta$-conglycinine native & $-0,92^{c}$ & $-0,79^{a}$ & $-0,52$ & $0,83^{\mathrm{b}}$ \\
\hline
\end{tabular}

${ }^{1}$ DANR : digestibilité apparente de l'azote du régime ; DANS : digestibilité apparente de l'azote du soja ; GPV : gain de poids vif ; TAAS : titre d anticorps anti-soja. ${ }^{2} \mathrm{SH}:$ sérum hyperimmun, IHP : inhibition de l'hémagglutination passive. ${ }^{a} p<0,05,{ }^{b} p<0,01,{ }^{c} p<0,001$.

la matière sèche. La cuisson à la vapeur pendant 20 à 40 min permet d'inactiver la majeure partie de l'activité antitrypsique et des lectines, mais aussi de réduire les activités antigéniques de 80 à $90 \%$ par rapport aux produits crus (Toullec et al, 1994b ; Lallès et al, 1996b). Les traitements hydroéthanoliques réalisés dans des conditions opti- males (chauffage entre 55 et $76{ }^{\circ} \mathrm{C}$ pendant 2 h dans un mélange comprenant 70 à $80 \%$ d'éthanol) sont maintenant bien connus pour reduire beaucoup plus fortement les concentrations de globulines immunoréactives (Sissons et al, 1982). Enfin, la protéolyse (dans des conditions non dévoilées par les fabricants) présente les mêmes avantages et per- 
met d'améliorer le mieux la digestibilité de l'azote du soja qui est alors proche de celle observée avec le lactosérum (Lallès et al, 1995c). Ces deux derniers types de technologies ont été développés car les produits traités thermiquement, généralement satisfaisants pour alimenter les porcs et les volailles, sont mal digérés par le veau préruminant (digestibilité de l'azote de 0,50 à 0,60 , contre 0,92 à 0,95 pour la poudre de lait écrémé), et induisent des réactions d'allergie digestive parfois fatales (Sissons, 1982). Il importe cependant de pouvoir contrôler les activités antigéniques résiduelles des produits commerciaux.

Les relations entre activité antigénique de l'aliment et sensibilisation au soja étant établies (Kilshaw et Sissons, 1979 ; Lallès et al, 1996a), il était nécessaire d'améliorer le mode d'expression de l'activité antigénique et de mieux définir les modalités de dosage immunochimique des protéines antigéniques. En effet, les résultats ont été le plus souvent exprimés semi-quantitativement sous forme de titre (Kilshaw et Sissons, 1979 ; Sissons et al, 1982). De plus, des discordances entre dosages Elisa et IHP sur quelques échantillons ont conduit certains auteurs (revue de Huisman, 1989) à préférer l'Elisa, sans autre raison établie. Les études que nous avons entreprises depuis quelques années ont débouché sur les observations suivantes: l'expression des résultats sous forme de titre dépend fortement du sérum hyperimmun spécifique employé alors qu'une expression quantitative (en $\mathrm{mg} / \mathrm{g}$ MA) réduit les différences (Tukur et al, 1994) ; l'emploi d'anticorps monoclonaux en Elisa aboutit à des relations de prévision de la digestibilité beaucoup moins bonnes qu'avec les sérums hyperimmuns correspondants (Tukur et al, 1996). Les résultats présents confirment indirectement ces observations, en indiquant que le type de dosage (Elisa, IHP ou immunotache) affecte peu le classement des produits du soja (même si l'immunotache avec des globulines natives donne des concentrations plus élevées) et la qualité des relations entre paramètres in vivo et in vitro (tableau VI).

Les comparaisons entre ces trois dispositifs immunochimiques sont intéressantes pour plusieurs raisons. L'Elisa est souvent considérée comme la méthode de référence car elle est sensible, reproductible et automatisable. En revanche, elle nécessite un équipement spécifique (plaques, laveur de plaques, lecteur optique), parfois coûteux et peut-être délicat à utiliser dans des conditions de terrain. L'IHP a été choisie car elle a été beaucoup utilisée dans le passé (Kilshaw et Sissons, 1979 ; Sissons et al, 1982). Son inconvénient majeur est la préparation des globules rouges recouverts de protéines antigéniques, étape fastidieuse nécessitant l'emploi répété d'une centrifugeuse. L'amélioration de l'hémagglutination passive pourrait passer par l'emploi de billes de latex activées (Maehara et al, 1990). Enfin, la technique de l'immunotache, apparue dans les années 80 (revue de Stott, 1989) est sensible et simple à mettre en oeuvre (Berger et al, 1985). Elle ne nécessite pas d'équipement particulier car les dépôts peuvent être faits à l'aide d'une pipette sur les membranes ; de ce fait, elle paraît adaptée à des applications de terrain, même si la quantification des immunotaches est techniquement réalisable à l'aide d'un scanner et d'un ordinateur (Velleman, 1995). Enfin, les différences entre Elisa ou HIP et immunotache pourraient résulter des différences de « montage » des tests : dans les deux premiers cas, il s'agissait de dosages indirects par compétition et, dans le dernier, d'un dosage direct.

Le calcul des seuils de détection des protéines antigéniques par nos divers types de dosages indique la supériorité de l'Elisa (tableau VII). Les limites de détection de l'IHP et de l'immunotache semblent dépendre fortement de la protéine dosée. Ainsi le seuil de détection de l'IHP est bien meilleur lorsque le sérum hyperimmun antiglycinine dénaturée est utilisé. Enfin, celui 
Tableau VII. Limites de détection $(\mu \mathrm{g} / \mathrm{mL})$ de la glycinine et de la $\beta$-conglycinine immunoréactives selon le type de dosage immunochimique utilisé.

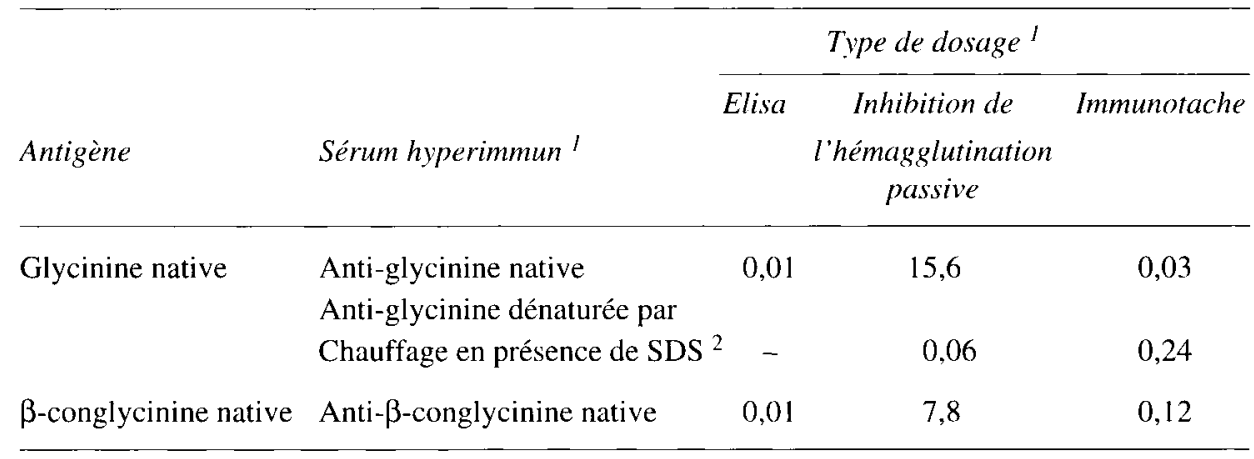

${ }^{1}$ Pour plus de détails, consulter le tableau II. ${ }^{2}$ SDS : dodécyl-sulfate de sodium $1 \%$, chauffage pendant 3 min.

de l'immunotache est généralement intermédiaire entre celui de l'Elisa et celui de l'IHP, et il est variable en fonction de l'antigène et du sérum hyperimmun employés.

Au-delà des dosages immunochimiques employés, il est important de noter que les coefficients de corrélation entre les paramètres in vivo et les critères analytiques in vitro sont le plus souvent élevés et significatifs dans le cas de la digestibilité de l'azote (du régime ou du soja) et de l'immunogénicité (titre d'anticorps anti-soja dans le plasma ; tableau VI) ; en revanche, ils sont moins élevés et ne sont significatifs qu'une fois dans le cas du GPV. Cela provient vraisemblablement du fait que la mesure d'antigénicité in vitro rend assez bien compte, d'une part, de l'état de dénaturation des protéines d'un produit du soja et de leur susceptibilité à être digérées et, d'autre part, de leur pouvoir immunogène. Le GPV est un critère plus synthétique qui n'est pas déterminé uniquement par la digestibilité de l'azote mais également par l'utilisation de l'ensemble des nutriments, dont les apports ont pu varier entre les essais conduits sur 3 ans. Il paraît donc assez logique qu'un critère analytique de dénaturation des protéines ne puisse pas rendre compte de la croissance des animaux avec une précision aussi grande que celle observée pour la digestibilité de l'azote ou l'immunogénicité.

\section{CONCLUSION}

Malgré le nombre limité d'échantillons analysés dans cette étude, les divers types de tests immunochimiques mis en oeuvre pour doser la glycinine et la $\beta$-conglycinine dans les dérivés du soja conduisent, le plus souvent, à des relations très significatives avec les paramètres in vivo que sont la digestibilité de l'azote des régimes ou des sources de soja et la production systémique d'anticorps spécifiques. Cela renforce l'idée qu'il faut privilégier l'emploi de sérums hyperimmuns spécifiques (dirigés contre les antigènes natifs ou dénaturés par chauffage en présence de SDS) par rapport à des anticorps monoclonaux, malgré la possibilité de produire ces derniers en grande quantité et avec une qualité définie. Cependant, l'intérêt des anticorps monoclonaux sera à réévaluer pour les produits dépourvus d'immunoréactivité $\beta$-conglycinine détectable à l'aide d'un sérum hyperimmun, et ayant des activités antitrypsiques résiduelles 
voisines (Lallès et al, 1996). Enfin, quelques différences, difficiles à interpréter car les montages immunochimiques n'étaient pas les mêmes, ont été observées entre certains tests.

Au plan pratique, la technique Elisa reste la méthode de choix dans les conditions du laboratoire ; l'inhibition de l'hémagglutination passive et l'immunotache pourront être choisies lorsque certains équipements font défaut ou sont trop délicats pour une utilisation sur le terrain.

\section{REMERCIEMENTS}

Nous remercions le personnel technique du laboratoire pour le travail sur les animaux, ainsi que Jeannine Quillet pour la recherche bibliographique. Nous remercions aussi le ministère des Affaires étrangères, le ministère des l'Agriculture et de la Pêche (contrat DGER n ${ }^{\circ}$ 91-131), la région Bretagne, le département d'Ille-et-Vilaine et le District de Rennes, pour leur participation financière à ces travaux.

\section{RÉFÉRENCES}

Berger PH, Thornbury DW, Pirone TP (1985) Detection of picogram quantities of potyviruses using a dot blot immunobinding assay. $J$ Virol Methods $12,31-39$

Boulard C, Lecroisey A (1982) Specific antisera produced by direct immunization with slices of polyacrylamide gel containing small amounts of protein. J Immunol Methods 50, 221-226

Carter JM, Lce HA, Mills ENC, Lambert N, Chan HWS, Morgan MRA (1992) Characterisation of polyclonal and monoclonal antibodies against glycinin (1IS storage protein) from soya (Glycine max). I Sci Food Agric 58, 75-82

Dagnélie $\mathrm{P}(1970)$ Les méthodes relatives à la régression. In : Théorie et Méthodes statistiques. Duculot, Gembloux, 395-398

Heppell LMJ, Sissons JW, Pedersen HE (1987) A comparison of the antigenicity of soya-based infant formulas. Br J Nutr 58, 393-403

Hollander M, Wolfe DA (1973) Multiple comparisons. In : Non Parametric Statistical Methods, Wiley \& Sons, New York. $115-334$

Huisman J (1989) Antinutritional factors (ANFs) in the nutrition of monogastric farm animals. In :
Nutrition and Digestive Physiology in Monogastric Farm Animals (EJ Van Weerden, J Huisman, eds) Pudoc, Wageningen, 17-35

Kilshaw PJ, Sissons JW (1979) Gastrointestinal allergy to soyabean protein in preruminant calves. Allergenic constituents of soyabean products. Res Vet Sci $27,366-371$

Lallès JP, Toullec R (1996) Digestion des protéines végétales et hypersensibilité digestive chez le veau préruminant. Inra Prod Anim 9, 255-264

Lallès JP, Tukur HM, Toullec R (1995a) Évaluation de l'antigénicité des produits du soja destinés aux aliments d'allaitement pour veaux : quels tests immunochimiques employer? Renc Rech Ruminants 2, 135

Lallès JP, Toullec R, Bouche $z$ P, Roger L (1995b) Antigenicity and digestive utilization of four soyabean products by the preruminant calf. Livest Prod Sci 41, 29-38

Lallès JP, Toullec R, Branco Pardal P, Sissons JW (1995c) Hydrolyzed soy protein isolate sustains high nutritional performance in veal calves. $J$ Dair Sci 78, 194-204

Lallès JP, Dréau D, Salmon H, Toullec R (1996a) Identification of soya bean allergens and immune mechanisms of dietary sensitivities in preruminant calves. Res Vet Sci 60, 111-116

Lallès JP, Tukur HM, Toullec R, Miller BG (1996b) Analytical criteria for predicting apparent digest $i$ bility of soy protein in preruminant calves. J Dairy Sci $79,475-484$

Maehara T, Eda Y, Mitani K, Matsuzawa S (1990) Methacrylate-styrene copolymer latex particles for immunologic agglutination tests. Biomaterials 11 . 122-126

Murphy PA, Resurreccion AP (1984) Varietal and environmental differences in soybean glycinin and $\beta$-conglycinin content. J Agric Food Sci 32, 911915

Nielsen SS, Deshpande SS, Hermodson MA, Scott MP (1988) Comparative digestibility of legume storage proteins. J Agric Food Chem 36, 896-902

Plumb GW, Mills ENC, Tatton MJ, D'Ursel CCM, Lambert N, Morgan MRA (1994) Effect of thermal and proteolytic processing on glycinin, the $11 \mathrm{~S}$ globulin of soy (Glycinine max): a study utilizing monoclonal and polyclonal antibodies. J Agric Food Chem 42, 834-840

Plumb GW, Lambert N, Mills ENC, Tatton MJ, D'Ursel CCM, Bogracheva T, Morgan MRA (1995) Characterisation of monoclonal antibodies against $\beta$-conglycinin from soy bean (G/ycinine $m a x$ ) and their use as probes for thermal denaturation. J Sci Food Agric 67, $511-520$

Sissons JW (1982) Effects of soya-bean products on digestive processes in the gastrointestinal tract of preruminant calves. Proc Nutr Soc 41, 53-61 
Sissons JW, Thurston SM (1984) Survival of dietary antigens in the digestive tract of calves intolerant to soyabean products. Res Vet Sci 37, 242-246

Sissons JW, Nyrup A, Kilshaw PJ, Smith RH (1982) Ethanol denaturation of soya-bean protein antigens. J Sci Food Agric 33, 706-710

Stott DI (1989) Immunoblotting and dot blotting. J Immunol Methods 119, 153-187

Toullec R, Lallès JP, Bouchez P (1994a) Substitution of skim milk protein with soyabean protein concentrates and whey in milk replacers for veal calves. Anim Feed Sci Technol 50, 101-112

Toullec R, Lallès JP, Tukur HM (1994b) Caractéristiques biochimiques et digestibilité apparente des matières azotées du soja chez le veau préruminant. Renc Rech Ruminants 1, 229-232

Tukur HM, Lallès JP, Mathis C, Caugant I, Toullec R (1993) Digestion of soya-bean globulins, glycinin, $\alpha$-conglycinin and $\beta$-conglycinin in the preruminant and the ruminant calf. Can J Anim Sci 73,891905
Tukur HM, Lallès JP, Toullec R (1994) Some aspects of the in vitro determination of antigenic activity in soyabean products for young calves. In : The Developing of Digestive and Metabolic Processes in New Born and Growing Ruminants (W Barej, $\mathrm{R}$ Zabielski, P Ostaszewski, eds) Proceedings of Satellite Symposium to the 8th International Symposium on Ruminant Physiology, Varsovie, 94-98

Tukur HM, Branco Pardal P, Formal M, Toullec R, Lallès JP, Guilloteau P (1995) Digestibility, blood levels of nutrients and skin responses of calves fed soyabean and lupin proteins. Reprod Nutr Dev 35 , $27-44$

Tukur HM, Lallès JP. Plumb GW, Mills ENC, Morgan MRA, Toullec R (1996) Investigation of the relationship between in vitro Elisa measures of immunoreactive soy globulins and in vivo effects of soy products. J Agric Food Chem 44, 2155-2161

Velleman SG (1995) Quantifying immunoblots with a digital scanner. Biotechniques 18, 1056-1058 